Relations industrielles

Industrial Relations

\title{
Le mouvement des grèves en France, par R. Goetz-Girey, Coll. " Economique ", no 3, Editions Sirey, Paris 1965, 217 pages.
}

\section{Pierre Dionne}

Volume 22, numéro 2, 1967

URI : https://id.erudit.org/iderudit/027795ar

DOI : https://doi.org/10.7202/027795ar

Aller au sommaire du numéro

Éditeur(s)

Département des relations industrielles de l'Université Laval

ISSN

0034-379X (imprimé)

1703-8138 (numérique)

Découvrir la revue

Citer ce compte rendu

Dionne, P. (1967). Compte rendu de [Le mouvement des grèves en France, par R. Goetz-Girey, Coll. «Economique », no 3, Editions Sirey, Paris 1965, 217 pages.] Relations industrielles / Industrial Relations, 22(2), 303-304.

https://doi.org/10.7202/027795ar

Tous droits réservés @ C Département des relations industrielles de l'Université Laval, 1967
Ce document est protégé par la loi sur le droit d'auteur. L'utilisation des services d'Érudit (y compris la reproduction) est assujettie à sa politique d'utilisation que vous pouvez consulter en ligne.

https://apropos.erudit.org/fr/usagers/politique-dutilisation/ 
Collective Bargaining in the United States Federal Civil Service, by Willem B. Vosloo, Public Personnel Association, Chicogo, Illinois, 1966, 226 pages.

Ce trovail d'abord entrepris ò une fin spécifique soit une thèse de doctorat, s'avéro d'une importance telle pour le * Public Personnel Association des Etats-Unis, qu'on décida de le publier. Ce qui caractérise surtout cette étude sur les relations de travail dons les services fédéraux aux Etots-Unis, et ce qui la rend si valable aux yeux de l'Association du Personnel, c'est qu'elle n'est pas basée que sur des recherches antérieures mais sur plus de cent entrevues ovec les dirigeants et les employés.

Ce livre met en évidence toute l'importance qu'a prise le syndicalisme dans le secteur public; nouvelle réalité pour des gouvernements qui devront désormais agir comme les autres employeurs et même en devenir le modèle. Aux Etats-Unis, les problèmes devenaient d'une acuité telle que le président Kennedy a voulu y remédier por une loi en 1962. Cette loi al'Executive Order 10,988 » est l'objet principal de ce volume.

Avant de traiter de la loi, l'auteur dans son deuxième chapitre attaque la source même des frictions entre les deux antagonistes en se référant d diverses causes comme la relation entre le droit des employés et l'idéologie démocratique, ou encore celle des formes de direction et la participation D'aspect théorique ce chapitre situe très bien les trois partis, le syndicat, le gouvernement et le public. Les différents problèmes soulevés sont la souveraineté de l'Etat, le partage des pouvoirs (Président, Congrès et directeur du personnel) et leur délégation, la bureaucratie, l'administration, les droits des trovailleurs (formes d'association, grève) et le bien public. Donc un chapitre d'une importance copitale.

Le reste du volume s'attache directement ou programme Kennedy. On y retrouve les différents efforts législatifs pour parvenir à lc reconnaissance syndicale dans la fonction publique, ouxquels efforts se joignent les différentes oppositions ainsi que les pressions politiques pour parvenir à la création par le Président Kennedy d'un Comité de Travail qui soumet en 1961 des recommandations sur lesquelles sero construit "I'Executive Order 10,988

Les pages qui suivent ont pour sujet l'application du programme. On en voit les phases principales et les diverses difficultés rencontrées, celles-ci, dues en grande partie dit-on à l'apothie des dirigeants, font l'ob- jet d'un chapitre complet Suivent ensuite les solutions à ces problèmes apportées par des organismes spécialement mis sur pied pour cette période difficile de transition: de plus on donne la répartition des employés en syndiqués et non syndiqués, les méthodes de négociation et la réaction des deux partis.

En conclusion, après avoir fait le point sur les différentes théories et idéologies mentionnées plus haut, l'auteur tente une projection et croit qu'il y aura peu de changement dans les nouvelles façons de procéder et cela pour quelque temps.

En somme, c'est un ouvrage complet et d'actualité. II faut le consulter pour être au courant de l'évolution des relations de travail chez les fonctionnaires.

\section{Normand LECUYER}

Le mourement des grèves en France, par R. Goetz-Girey, Coll. "Economique », no 3, Editions Sirey, Paris 1965, 217 pages.

a Les perturbations du marché du travail dues oux grèves et aux alock-out ont été souvent étudiés en France dans leurs aspects juridiques et sociologiques $»$.

Cependant, les aspects économiques des grèves, leur description, leurs composantes, leurs causes et leurs effets, n'ont été étudiés de façon systématique que par un petit nombre d'auteurs parce que l'enquête statistique est malaisée et discontinue en France.

Malgré ces difficultés, l'auteur nous livre quelques résultats d'une recherche d'ensemble sur les grèves en France depuis 1919. Cette étude comporte quatre chapitres.

Le premier décrit l'environnement dans lequel se situe le mouvement des grèves tandis que le deuxième tente de saisir le mouvement des grèves dans ses diverses composantes pour la France entière, par régions et par secteurs industriels. Dans le troisième, l'auteur essaie de déterminer les facteurs qui exercent une influence sur lo propension ò la grève. Enfin, le dernier chapitre s'attache aux incidences et oux résultats des grèves.

En effet, les grèves peuvent avoir des effets directs ou indirects, limités ou très étendus. S'il est relativement facile de savoir quelles pertes de salaires ont éprouvé les grévistes, il est beaucoup plus difficile de préciser quelles sont les commandes perdues par l'entreprise en grève et la désutilité ressentie par les consommateurs. II est plus 
malaisé encore de donner des chiffres si I'on se place au niveau de l'économie nationale.

Si l'on reprend l'analyse menée par Chamberlain, on constatera avec l'auteur que sept groupes sont atteints por la grève:

1.-les individus ou institutions privées qui consomment les produits ou utilisent les services de l'entreprise en grève;

2.--les grévistes eux-mêmes et leurs familles dans la mesure où ils ne touchent plus de solaires;

3.-les producteurs directs qui rendent des services et vendent des biens oux grévistes et à leurs fomilles;

4.-les utilisateurs des produits de la firme en grève, qui ne peuvent plus s'approvisionner ;

5.-les producteurs indirects qui vendent des produits aux fournisseurs et aux utilisateurs de la firme.

6.-les consommoteurs de produits fournis par les producteurs indirects.

7.-l'Etat, si l'intérêt national est compromis par la grève.

Ainsi, les incidences d'une grève sont donc extrêmement vastes et les répercussions en chaîne débordent le cadre de la firme.

Cet ouvroge tend à nous démontrer par I'analyse statistique que les jugements portés sur les grèves ne dépendent pas uniquement des valeurs économiques; ils dépendent avant tout du monde des valeurs auxquelles les uns et les autres nous nous référons.

\section{Pierre DIONNE}

\section{Syndicalisme en péril ? La leçon américaine,} par B.J. Widick, traduit de l'anglais par C. Convers et Annie Gérard, Coll. «Masses et Militants $»$, Les Editions Ouvrières Poris, 1966, 215 pages.

Cette publication, dont l'édition originale est parue chez Houghton Mifflin Co., Boston, U.S.A., sous le titre Labor Today, s'inscrit dans un effort des milieux français pour saisir l'essentiel du syndicalisme sous ses différentes formes. La colection Masses et Militants $»$, en publiant ce volume de B.J. Widick, vient s'enrichir d'un point de vue nouveau et original sur le syndicalisme oméricoin.

L'auteur a vécu près de trente ans à I'intérieur du mouvement ouvrier à titre de spécicliste, conseiller ou rechechiste. II se considère lui-même comme un intellectuel du syndicalisme. Sa perspective est criginale cor, loin de se faire un défenseur acharné du syndicalisme contre ses nombreux ennemis, M. Widick tente plutôt de cerner les problèmes qui assiègent le syndicalisme, en n'hésitant pas à dénoncer les tores particulières cux syndicats américains. L'importance de ces associations l'amène à des considérations sur l'avenir de la société américaine et la survie de so forme de démocrotie.

Lo première partie du volume vient illustrer l'impuissance du syndicalisme américain à maîtriser certaines conséquences de l'automation. Cette analyse a le grand mérite de ne pas être seulement théorique. A grand renfort de données factuelles, de déclarations de personnages importants ou d'ouvriers, de statistiques ou d'articles de revues spécialisćes, l'auteur nous fait voir de l'intérieur les problèmes du chômage, des mises à pied spectoculaires (Ex: industries de l'automobile et de l'acier), des changements dans les structures de l'emploi et de la répugnance des «cols blancs » envers l'action syndicale, et enfin ceux de l'écart de plus en plus grand existant entre la base et la hiérarchie syndicale. Dans une deuxième partie, il vient creuser encore plus ces problèmes. Analysant les éléments qui peuvent être les causes du discrédit dans lequel baigne le syndicalisme américain actuel, il relève entre autres: absence de dynamisme, bureaucratie poussée à l'extrême, corruption, embourgecisement, etc. Sa réflexion touche ensuite le système des conventions collectives, dont il démasque plusieurs déficiences. II pose crûnent la question: ce système rapporte-t-il ce qu'on doit en attendre?

Les trois chapitres suivants constituent l'essentiel de la publication. II s'agit d'une réflexion sur la démocratie et les valeurs syndicales, sur l'isolement des intellectuels et sur le déclin du pouvoir politique. Encore une fois, la théorie n'est ici que l'accessoire; I'auteur s'oriente surtout vers une analyse factuelle. Dans l'interprétation des faits, on sent évidemment que l'auteur a «sa » conception du syndicalisme et que celle-ci influence son jugement, lui donne une colorotion particulière. Que les conceptions d'un auteur transpirent dans ses écrits, cela est tout à fait normal. II nous semble cependant que l'ouvrage y aurait gagné en netteté et précision si l'auteur s'était obligé à dégager rapidement les éléments de sa conception.

Nous soulignons les mérites de cette traduction, mais le titre donné par les éditeurs français nous porte à sourire. II nous apparaît comme une monifestation du manque 